\section{Review: group-based behavioural and cognitive-behavioural parenting interventions are effective and cost- effective for reducing early-onset child conduct problems}

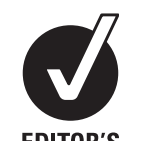

\section{QUESTION}

Question: Are group-based behavioural and cognitive-behavioural parenting interventions EDITOR'S effective and cost-effective in reducing early-onset CHOICE child conduct problems?

Outcomes: Primary outcomes: child behaviour; parental skills; parental mental health; cost-effectiveness. Effects on shortterm outcomes (up to 3 months post-treatment) were assessed.

\section{METHODS}

Design: Systematic review and meta-analysis.

Data sources: CENTRAL (2011, Issue 1), MEDLINE (1950 onwards), EMBASE (1980 onwards), CINAHL (1982 onwards), PsycINFO (1872 onwards), Social Science Citation index (1956 onwards), ASSIA (1987 onwards), ERIC (1966 onwards), Sociological Abstracts (1963 onwards), Academic Search Premier (1970 onwards), Econlit (1969 onwards), PEDE (1980 onwards), Dissertations and Theses Abstracts (1980 onwards), NHS EED (searched 31 January 2011), HEED (searched 31 January 2011), DARE (searched 31 January 2011), HTA (searched 31 January 2011) and randomised controlled trials (mRCT) (searched 29 January 2011) were searched in January 2011. The following parent training websites were searched on 31 January 2011: Triple P Library, Incredible Years Library and Parent Management Training. The reference lists of studies and reviews were hand searched.

Study selection and analysis: RCTs or quasi-RCTs of behavioural and cognitive-behavioural group-based parenting interventions for parents of children aged 3-12 years with conduct problems were included. The control group had to be a waiting list, no treatment or standard treatment; and the study had to use at least one standardised instrument for measurement of child conduct problems for inclusion. Meta-analysis was carried out using RevMan 5.1 software. Mean effect sizes for continu- ous outcomes were pooled using the generic inverse variance methods to give the standardised mean difference (SMD). RRs for dichotomous outcomes were converted into SMDs. Random effects methods were used due to heterogeneity in the analyses. Effect sizes were interpreted as providing 'no evidence of effectiveness' if $\mathrm{SMD}<0.20$, 'small' if $0.20<\mathrm{SMD}<0.40$, 'moderate' if $0.40<\mathrm{SMD}<0.75$, 'large' if $>0.75$.

\section{MAIN RESULTS}

Thirteen trials (1078 participants) and two economic evaluations based on two of the trials were included in the review. According to parental and independent assessment, parent training interventions significantly reduced child conduct problems, improved positive parenting practices and reduced negative parental practices compared with control (please refer Webextra table). Parent training programmes were also found to improve parental mental health significantly with small to moderate effect sizes and no heterogeneity between studies (eight studies; $\mathrm{n}=636$; $\mathrm{SMD}-0.36,95 \% \mathrm{CI}-0.52$ to -0.20 ; $\mathrm{p}<0.0001)$. Both of the economic evaluations assessed the Incredible Years' Parenting programme compared with treatment as usual. The overall mean cost per parent, presented in 2011 international dollar (\$) values, was \$3407.51 for one of the economic evaluations undertaken in 2007 and $\$ 1615.22$ for the other study, undertaken in 2011. The two studies demonstrated evidence of cost-effectiveness of parenting interventions. It was calculated that it would cost $\$ 2368.53$ (the 2007 study) and \$2464.24 (the 2011 study) to bring the average child with clinical levels of conduct problems into the non-clinical range.

\section{CONCLUSIONS}

Behavioural and cognitive-behavioural group-based parenting intervention programmes are effective and cost-effective for improving early-onset child conduct problems, parental mental health and parenting skills in the short term. Further research is needed on the long-term outcomes of these programmes.

\section{ABSTRACTED FROM}

Furlong M, McGilloway S, Bywater T, et al. Behavioural and cognitive-behavioural group-based parenting programmes for early-onset conduct problems in children aged 3 to 12 years. Cochrane Database Syst Rev 2012;2:CD008225.

Correspondence to: Mairead Furlong, Department of Psychology, John Hume Building, National University of Ireland Maynooth, Maynooth, Co Kildare, Ireland; mairead.m.furlong@nuim.ieCorrespondence to: Mairead Furlong, Department of Psychology, John Hume Building, National University of Ireland Maynooth, Maynooth, Co Kildare, Ireland; mairead.m.furlong@nuim.ie

Sources of funding The Health Research Board of Ireland, and National University of Ireland Maynooth.
Furlong et al conclude that group-based behavioural and cognitive-behavioural parenting interventions are effective for improving children's conduct problems, and parents' parenting skills and mental health. So what's new, when many earlier reviews have drawn similar conclusions? First, this review asks a clearlyfocused clinical question, and is conducted to an impressive Cochrane level of thoroughness and rigour. Second, in a field that is moving rapidly from developer-led efficacy trials, to implementation trials in complex realworld services, we need to be sure that effect sizes are not declining over time, as developer control over trials is relaxed. Lower effect sizes tend to result from improved standards of trial conduct and reporting. This review sheds new light on both these issues, using a careful series of sensitivity analyses. Reassuringly, and in contrast to findings from other fields, the authors neither found decline in effect sizes with increasing study quality nor were the effect sizes stronger when the programme developer conducts the trial. Although other aspects of the efficacy versus effectiveness dimension are not investigated, the findings are striking in suggesting that parenting interventions are effective across a wide range of countries, 'real-world' service settings and families. Examining findings from this review and other high-quality studies (some excluded as they focus on targeted prevention), we can conclude that parenting interventions appear to be effective for families with high and low levels of deprivation, with and without maternal depression, those from ethnic minorities and majorities and those with severe and moderate levels of conduct problems. The question of age effects is not addressed. Of course, basic science tells us younger children will benefit more from intervention. Yet surprisingly, some intervention reviews suggest the opposite: this needs up-to-date synthesis.

Overall, this review shows it is feasible, and likely to be cost effective, to take these beneficial intervention effects into real-world services. It affirms the continuation of sterling efforts to go 'to scale' (eg, in the UK by the Improving Access to Psychological Therapies Programme), but this requires careful attention to practitioner fidelity, training and supervision, as well as high levels of political and professional enthusiasm.

\section{Frances Gardner}

Professor of Child and Family Psychology, Department of Social Policy and Intervention, Oxford University, UK

Competing interests None. 